\title{
IPRT/AMATERAS: A New Metric Spectrum Observation System for Solar Radio Bursts
}

\author{
K. Iwai · F. Tsuchiya $\cdot$ A. Morioka $\cdot$ H. Misawa
}

Received: 5 July 2011 / Accepted: 2 December 2011 / Published online: 3 January 2012

(C) The Author(s) 2011. This article is published with open access at Springerlink.com

\begin{abstract}
A new radio spectropolarimeter for solar radio observation has been developed at Tohoku University and installed on the Iitate Planetary Radio Telescope (IPRT) at the Iitate observatory in Fukushima prefecture, Japan. This system, named AMATERAS (the Assembly of Metric-band Aperture TElescope and Real-time Analysis System), enables us to observe solar radio bursts in the frequency range between 150 and $500 \mathrm{MHz}$. The minimum detectable flux in the observation frequency range is less than $0.7 \mathrm{SFU}$ with an integration time of $10 \mathrm{~ms}$ and a bandwidth of $61 \mathrm{kHz}$. Both left and right polarization components are simultaneously observed in this system. These specifications are accomplished by combining the large aperture of IPRT with a high-speed digital receiver. Observational data are calibrated and archived soon after the daily observation. The database is available online. The high-sensitivity observational data with the high time and frequency resolutions from AMATERAS will be used to analyze spectral fine structures of solar radio bursts.
\end{abstract}

Keywords Radio bursts $\cdot$ Instruments $\cdot$ Antenna $\cdot$ Radio spectrometer $\cdot$ Fast-Fourier transform spectrometer

\section{Introduction}

In the solar corona, there are many particle acceleration phenomena that are caused by the interactions between coronal magnetic field and plasma. Nonthermal electrons accelerated in the corona emit radio waves in the metric range, resulting in many types of observed solar radio bursts (see review of McLean and Labrum, 1985). Since there remain many unsolved processes for particle acceleration and radio emission of these solar radio bursts, monitoring of their observations is important in solar coronal physics.

K. Iwai $(\bowtie) \cdot$ F. Tsuchiya $\cdot$ A. Morioka $\cdot$ H. Misawa

Planetary Plasma and Atmospheric Research Center, Tohoku University, Sendai, Miyagi 980-8578,

Japan

e-mail: kazumasa-iwai@pparc.gp.tohoku.ac.jp

url: http://pparc.gp.tohoku.ac.jp/ 
There are many solar radio spectrum observation facilities in the world, such as Hiraiso in Japan (Kondo et al., 1995), Bleien in Switzerland (Messmer, Benz, and Monstein, 1999), Culgoora in Australia (Prestage et al., 1994), Tremonsdorf in Germany (Mann et al., 1992), and e-CALLISTO observatories (Benz et al., 2009a). As the observation technologies have improved, a variety of spectral fine structures have been observed in solar radio bursts such as spike bursts (Güdel and Benz, 1990; Magdalenic et al., 2006; Benz et al., 2009b), type I bursts (Sundaram and Subramanian, 2005 and references therein), and fiber bursts or zebras in type IV bursts (Aurass et al., 2003; Chernov, 2006). More detailed analyses of their spectral structures are thought to be important in understanding particle dynamics in the solar corona.

However, strong radio frequency interference (RFI) has been increasing in the metric range, and ground-based radio observation in this frequency band has become increasingly more difficult. It is difficult for wideband spectral observation in the metric range to avoid RFI, as the intensities are much stronger than those of any natural radio emissions. Saturations of the received signal in receivers and analyzers could be a serious problem. Even if the saturations are avoided, it is difficult to recognize spectral structures when a number of RFIs are present at the same frequencies.

Some technical problems remain in improving the resolution and sensitivity of solar radio observation. As the radio flux of the quiet Sun is very strong in the metric range and the antenna temperature is usually much greater than the noise temperature of the receivers, the sensitivity to detect bursty emission is limited by the bright solar disk emission. The antenna temperature is proportional to the effective aperture area of a radio telescope (see Equation (8) in Section 2) if the main beam width of the telescope is larger than the diameter of the Sun. Therefore, a large aperture of a radio telescope does not improve its sensitivity to a weak radio burst. However, a large aperture telescope increases the antenna temperature of the solar radio emissions only; it suppresses that of RFIs since almost all RFIs come from side lobes of the antenna beam. As a result, the larger aperture size telescope enables a wider dynamic range observation of radio emission from the Sun. The upper limit of the telescope's diameter is about $40 \mathrm{~m}$ (beam size of $1-2^{\circ}$ at $300 \mathrm{MHz}$ ) for monitoring observations of metric solar radio bursts since the solar disk is about $0.5^{\circ}$.

The sensitivity of frequency agile or swept-frequency systems is limited since the signal intensity in each frequency channel is measured sequentially. Acousto-optical spectrometers (AOSs) have sometimes been used for high-sensitivity observations of solar radio bursts (Benz, Messmer, and Monstein, 2001; Kontogeorgos et al., 2006), since AOS observations are simultaneous in all frequency channels. However, AOSs are relatively limited in dynamic range, and thus they are apt to be saturated by intense RFIs. Their stability and frequency channels are also insufficient to obtain a continuous high-dispersion spectrum. Fast Fourier transform (FFT) spectrometers using an analog-digital (A/D) converter also have been adopted for solar radio observation (see, e.g., Benz et al., 2009b). These instruments are able to maintain a high sensitivity in high time and frequency resolution observations with enough stability. Although the legacy A/D converters also had limited bandwidth due to their limited sampling speed, the digital technology has improved recently and the observation bandwidth has become ever wider. However, the commonly used A/D converters have not yet achieved a sufficient dynamic range to avoid saturation from RFIs (e.g., 8-bit A/D sampling attains a dynamic range of only $48 \mathrm{~dB}$ ).

These saturation problems can be solved by using a large aperture telescope that reduces RFIs. Therefore, it is suggested that the combination of a large aperture telescope and an A/D + FFT analyzer will enable us to observe metric solar radio emissions with higher resolution and higher sensitivity against RFIs. We have developed a new meter wave range solar 


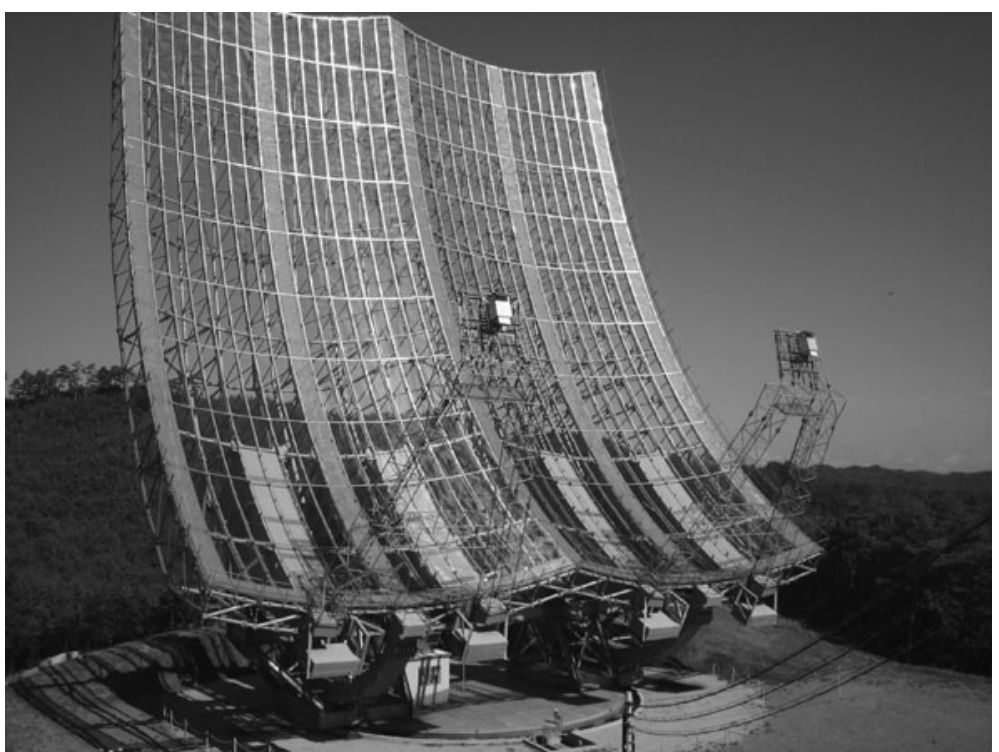

Figure 1 An overall view of the IPRT.

radio observation system using a $1023-\mathrm{m}^{2}$ aperture telescope, called the Iitate Planetary Radio Telescope (IPRT), and a spectro-polarimeter using an FFT spectrometer with an 8-bit A/D converter. The main beam of the IPRT is about $2^{\circ}$ at $300 \mathrm{MHz}$, which is suitable for monitoring solar radio bursts. This system, called AMATERAS, ${ }^{1}$ the acronym for Assembly of Metric-band Aperture TElescope and Real-time Analysis System, enables observation of solar radio bursts with high time and frequency resolutions and high sensitivity without any saturation by RFIs. The large aperture telescope also enables observation of several flux calibration sources to improve the system calibration, which is generally difficult for small aperture radio telescopes.

This paper reports on the new AMATERAS with some examples of the observed frequency spectrum of solar radio bursts. Section 2 gives an overview of the AMATERAS hardware and specifications, and Section 3 shows some radio burst observation results. Section 4 describes the database of the radio spectrograms observed by AMATERAS. Lastly, Section 5 summarizes and concludes the paper.

\section{Observation System}

\subsection{Iitate Planetary Radio Telescope (IPRT)}

IPRT is a ground-based radio telescope developed by Tohoku University. IPRT has been developed at the Iitate observatory in Fukushima prefecture, Japan, since 2000 and was originally used to observe Jovian radio emissions (Tsuchiya et al., 2010). Figure 1 shows an overall view of IPRT. IPRT is composed of two identically shaped rectangular offset parabolic sections. The reflecting surface is formed by fine-mesh stainless steel (pitch size:

\footnotetext{
${ }^{1}$ Amaterasu is a Sun goddess in Japanese mythology.
} 
Table 1 Specifications of the IPRT.

\begin{tabular}{ll}
\hline Parameter & IPRT \\
\hline Antenna type & Dual asymmetric offset parabola \\
Physical aperture & $1023 \mathrm{~m}^{2}(31 \mathrm{~m} \times 16.5 \mathrm{~m} \times 2$ sets $)$ \\
Focal length & $12 \mathrm{~m}(\mathrm{~F} / \mathrm{D}: 0.39)$ \\
Pointing accuracy & 0.1 \\
Reflecting surface & fine-mesh $($ pitch size: $20 \mathrm{~mm} \times 20 \mathrm{~mm})$ \\
\hline
\end{tabular}
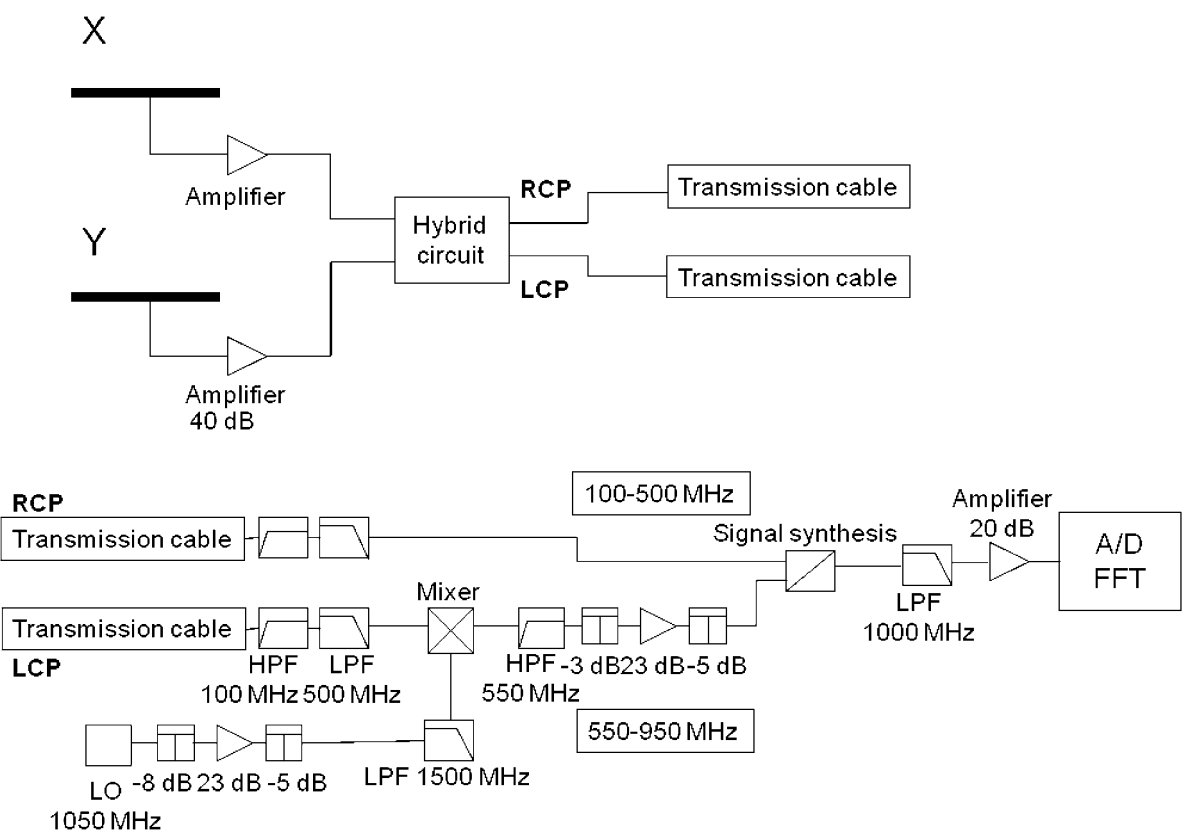

Figure 2 Block diagram of IPRT/AMATERAS.

$20 \mathrm{~mm} \times 20 \mathrm{~mm}$ ). The two rectangular parabolic sections are installed on one alt-azimuth mount and have one beam direction. Each parabolic section has the same physical aperture area of $511.5 \mathrm{~m}^{2}(16.5 \mathrm{~m} \times 31 \mathrm{~m})$, and the total physical aperture area is $1023 \mathrm{~m}^{2}$. The IPRT specifications are listed in Table 1. IPRT is programmed to track the Sun every 30 seconds from sunrise to sunset every day. Daily observation, data processing, and data archiving processes are all performed automatically.

\subsection{Solar Radio Observation System of IPRT (AMATERAS)}

Figure 2 shows the block diagram of the solar radio observation system of IPRT, AMATERAS. This system uses only one rectangular parabolic section of IPRT. Hence, the physical aperture of this system is $511.5 \mathrm{~m}^{2}$. At the focal point of IPRT, there is a plane reflector to form the directivity of the primary feed elements. Crossed dipole elements appropriate to detect radio waves from 200 to $300 \mathrm{MHz}$ are installed on the plane reflector. Each element is mounted at an angle of $45^{\circ}$ from the long axis of the rectangular parabolic section in order to detect the two orthogonal polarization components equally. 
A front-end receiver is installed behind the plane reflector. In the front-end receiver, signals received by the two orthogonal feed elements are amplified by $40 \mathrm{~dB}$ in preamplifiers and separated into left- and right-handed circularly polarized components (LCP and RCP) in a hybrid coupler. The LCP and RCP signals are sent to the back-end receiver system installed in a radio shielded room located on the turntable of IPRT through 66-m coaxial cables.

In the back-end receiver, signals lower than $100 \mathrm{MHz}$ and higher than $500 \mathrm{MHz}$ are filtered out. Then, the LCP signal is up-converted to a higher frequency range using the superheterodyne system, where the signals of 100 to $500 \mathrm{MHz}$ are converted to 950 to $450 \mathrm{MHz}$ by the local oscillator signal of $1050 \mathrm{MHz}$, and combined with the RCP signal in order to measure both LCP and RCP signals simultaneously using one frequency analyzer instrument. The isolation of the LCP and RCP signals of the receiver system including the polarization circuit and back-end system is about $26 \mathrm{~dB}$ at $200 \mathrm{MHz}$ and better than $20 \mathrm{~dB}$ in the full observational band from 100 to $500 \mathrm{MHz}$.

The combined LCP and RCP signals are finally A/D converted and broken into a power spectrum by FFT in an AC240 frequency analyzer (Benz et al., 2005). The AC240 board includes two A/D converters and one digital processing unit using a field programmable gate array (FPGA). The two A/D converters have a $1 \mathrm{GHz}$ sampling rate and are combined to form a single $2 \mathrm{GHz} 8$-bit converter. The FPGA unit performs FFT using $2^{14}$ (16384) frequency channels. Hence, the frequency resolution of this system is about $61 \mathrm{kHz}$. The spectra are obtained every $16384 \mu \mathrm{s}$. They are accumulated every $10 \mathrm{~ms}$ in the FPGA and output in a linear scale with 36-bit data length. The output spectrum has a dynamic range of about $60 \mathrm{~dB}$. The transfer rate of the accumulated data is less than $10 \mathrm{~ms}$, which means that there is no dead time in obtaining the spectrum.

The total system gain is set so as to avoid saturation by the strongest RFI at the observation site. The output signal level of the quiet Sun is 5-12 dB larger than the noise level of the receiver system in the observation band. The saturation level of this system is determined by the bandwidth of the input burst signal. It is about 20000 solar flux units (SFU) for the radio burst with a bandwidth of $300 \mathrm{MHz}$ and about $200000 \mathrm{SFU}$ for the radio burst with a bandwidth of $30 \mathrm{MHz}\left(1 \mathrm{SFU}=10^{-22} \mathrm{~W} \mathrm{~m}^{-2} \mathrm{~Hz}^{-1}\right)$.

\subsection{Calibration}

The calibration of AMATERAS consists of two steps. First, the total gain and noise temperature of the receiver are measured using the Y-factor method (see, e.g., Kraus, 1986). Second, the effective aperture area and minimum detectable flux of the entire observation system are measured by observing a calibration source. All system parameters depend on frequency; hence, the parameters are measured at each observation frequency channel.

The gain $(G)$ and noise temperature of the receiver system $\left(T_{\mathrm{RX}}\right)$ are measured using two calibrated noise signals whose equivalent temperatures are $T_{\text {hot }}$ and $T_{\text {cold }}$. When the input of the receiver is terminated by $T_{\text {hot }}$ and $T_{\text {cold }}$, the signal output powers, $P_{\text {hot }}$ and $P_{\text {cold }}$, are given as

$$
\begin{gathered}
P_{\mathrm{hot}}=k_{\mathrm{B}} G \Delta f\left(T_{\mathrm{RX}}+T_{\mathrm{hot}}\right), \\
P_{\text {cold }}=k_{\mathrm{B}} G \Delta f\left(T_{\mathrm{RX}}+T_{\text {cold }}\right),
\end{gathered}
$$

where $k_{\mathrm{B}}$ is Boltzmann's constant and $\Delta f$ is an observation bandwidth. $T_{\mathrm{RX}}$ and $G$ can be determined by

$$
T_{\mathrm{RX}}=\frac{T_{\mathrm{hot}}-Y T_{\mathrm{cold}}}{Y-1},
$$


Figure 3 Total system gain $G$ of left-handed and right-handed circular polarization (LCP and RCP). The solid line shows RCP; dotted line shows LCP.

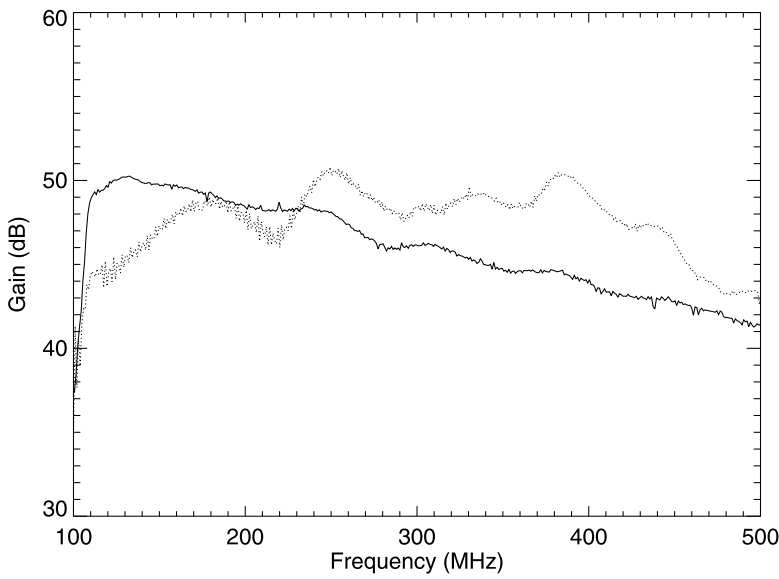

$$
G=\frac{P_{\text {hot }}}{k_{\mathrm{B}} \Delta f\left(T_{\text {hot }}+T_{\text {cold }}\right)},
$$

where $Y$ is a power ratio between $P_{\text {hot }}$ and $P_{\text {cold }}\left(Y=P_{\text {hot }} / P_{\text {cold }}\right)$.

The system gain $G$ of LCP and RCP are shown in Figure 3. The system gains of LCP and RCP at the same frequency are different because of the different signal paths, as shown in Figure 2. They are calibrated by the Y-factor sequence.

The effective aperture area and its dependence on the frequency are measured by the drift scan observation for a calibration source. The received power output from the back-end receiver during on $\left(P_{\text {cal }}\right)$ and off sources $\left(P_{\text {off }}\right)$ of the calibration source are given by

$$
\begin{gathered}
P_{\text {cal }}=k_{\mathrm{B}} G \Delta f\left(T_{\mathrm{RX}}+T_{\text {sky }}+T_{\mathrm{cal}}\right), \\
P_{\text {off }}=k_{\mathrm{B}} G \Delta f\left(T_{\mathrm{RX}}+T_{\text {sky }}\right),
\end{gathered}
$$

where $T_{\text {cal }}$ and $T_{\text {sky }}$ are the antenna temperatures for the calibration source and background sky, respectively. The brightness temperature of the calibration source is determined by

$$
T_{\text {cal }}=\frac{P_{\text {cal }}-P_{\text {off }}}{k_{\mathrm{B}} \Delta f G} .
$$

The effective aperture area of the radio telescope $\left(A_{\mathrm{e}}\right)$ is obtained as

$$
A_{\mathrm{e}}=\frac{2 k_{\mathrm{B}} T_{\mathrm{cal}}}{S}
$$

where $S$ is the flux density of the calibration source. Cassiopeia A or Cygnus A are used as the calibration sources; their flux densities are derived by referring to Baars et al. (1977). Figure 4 shows the observation result of Cassiopeia A at $200 \mathrm{MHz}$. The galaxy background is derived from the linear approximation of the flux before and after the on-source of the calibrator (linear solid line in Figure 4). The derived background is subtracted from the observed light curve in order to get the flux from the calibrator. In Figure $4, P_{\text {cal }}$ and $P_{\text {off }}$ are $6.32 \times 10^{-8} \mathrm{~mW}$ and $5.65 \times 10^{-8} \mathrm{~mW}$, respectively. $A_{\mathrm{e}}$ is calculated to be $259 \mathrm{~m}^{2}$ using the system gain at $200 \mathrm{MHz}(48.6 \mathrm{~dB})$.

The theoretical relative rms noise of the system is expected to be

$$
\frac{\Delta F}{F}=\frac{1}{\sqrt{\Delta f \tau}},
$$


Figure 4 Observational result of Cassiopeia A at $200 \mathrm{MHz}$. Linear solid line shows the galaxy background derived from the linear approximation of the flux before and after the on-source of the calibrator.

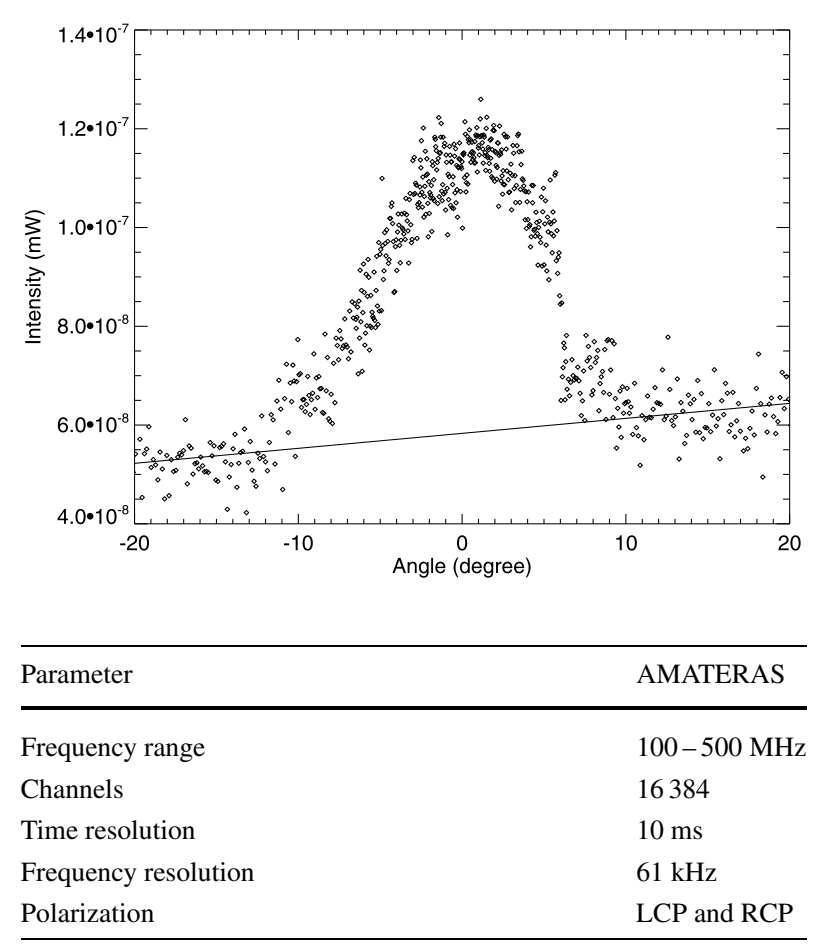

\begin{tabular}{ll}
\hline Parameter & AMATERAS \\
\hline Frequency range & $100-500 \mathrm{MHz}$ \\
Channels & 16384 \\
Time resolution & $10 \mathrm{~ms}$ \\
Frequency resolution & $61 \mathrm{kHz}$ \\
Polarization & $\mathrm{LCP}$ and RCP \\
\hline
\end{tabular}

Table 2 Specifications of solar observation system of the IPRT/AMATERAS.

Table 3 Frequency characteristics of calibration results of AMATERAS (RCP). Accumulation time is $10 \mathrm{~ms}$ and bandwidth is $61 \mathrm{kHz}$. Total system noise (the sum of $T_{\mathrm{RX}}$ and $T_{\text {sky }}$ ) is assumed to be $500-1500 \mathrm{~K}$.

\begin{tabular}{lllllll}
\hline Observation frequency $(\mathrm{MHz})$ & 160 & 230 & 300 & 380 & 420 & 470 \\
Total system gain $G(\mathrm{~dB})$ & 49.7 & 47.4 & 46.1 & 44.6 & 43.1 & 42.2 \\
Effective aperture area $A_{\mathrm{e}}\left(\mathrm{m}^{2}\right)$ & 31.4 & 158.3 & 186.3 & 83.6 & 69.4 & 72.3 \\
Sensitivity $\Delta S_{\min }(\mathrm{SFU})$ & 0.69 & 0.045 & 0.057 & 0.051 & 0.140 & 0.174 \\
\hline
\end{tabular}

where $F$ and $\Delta F$ are the mean flux and rms noise, respectively and $\tau$ is the accumulation time. The theoretical relative rms noise of this system is about 0.040 at a bandwidth of $61 \mathrm{kHz}$ and an accumulation time of $10 \mathrm{~ms}$. The observed relative rms noise at $200 \mathrm{MHz}$ was 0.039 .

The minimum detectable flux $\left(\Delta S_{\min }\right)$ of the system is given by

$$
\Delta S_{\min } \frac{2 k_{\mathrm{B}}\left(T_{\mathrm{RX}}+T_{\text {sky }}\right)}{A_{\mathrm{e}} \sqrt{\Delta f \tau}} .
$$

Tables 2 and 3 summarize specifications and calibration results of AMATERAS. This system performs well enough to observe solar radio bursts in the frequency range between 150 and $500 \mathrm{MHz}$. The minimum detectable flux in this frequency range is less than $0.7 \mathrm{SFU}$ with $10 \mathrm{~ms}$ accumulation time and $61 \mathrm{kHz}$ bandwidth. Simultaneous observations for both RCP and LCP are possible. Although the receiver bandwidth of this system is between 100 and $500 \mathrm{MHz}$, the sensitivity worsens steeply below $150 \mathrm{MHz}$ because of the frequency characteristics of the feed system. The feed elements will be revised in order to observe solar radio bursts in the full observation band of this system as a future work. 


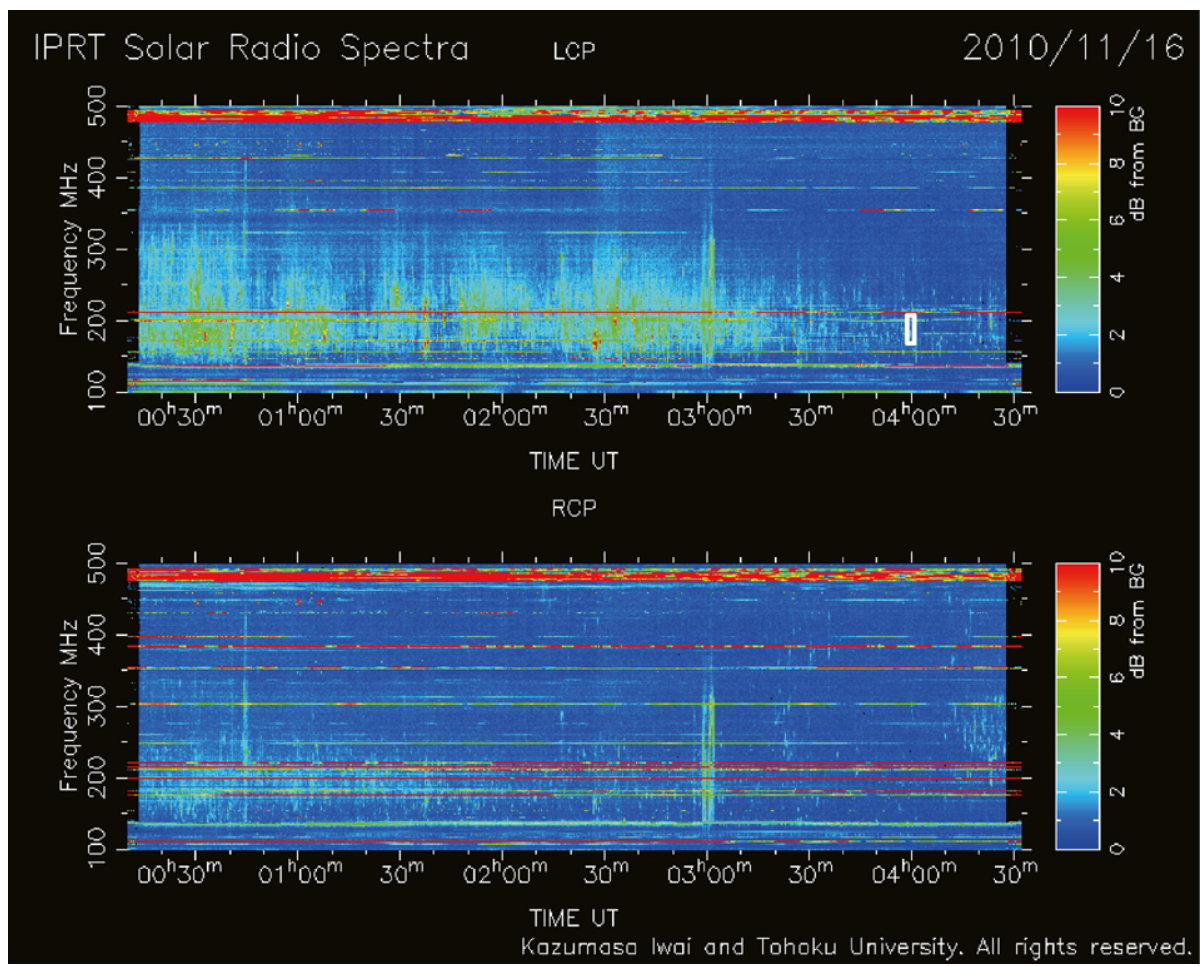

Figure 5 Radio dynamic spectra observed by IPRT on 16 November 2010: (top) LCP component and (bottom) RCP component.

\section{Observational Results}

IPRT/AMATERAS has been doing test observations of the Sun since September 2009, which is around the end of the solar minimum of Cycle 23 (or the early stage of Cycle 24). The first radio burst was detected in the C-class flare event occurring on 16 December, 2009. All systems were completely developed and installed by September 2010, and regular observations have been made since then.

\subsection{Polarization and High-Resolution Observation}

Figure 5 shows radio dynamic spectra observed with AMATERAS on 29 November 2010. Wideband flux enhancements were observed in both RCP and LCP around 3:00 UT. These emissions are type III bursts. Radio emissions that have only an LCP component appeared in the frequency range between 100 and $300 \mathrm{MHz}$ from 0:00 to 3:30 UT. These strongly polarized emissions are type I noise storms. Figure 6 shows a zoomed-in image of the spectrum indicated by the white rectangle in Figure 5. High-resolution observation of this system allows us to identify the radio bursts as a group of fine-spectrum structures with a duration of $0.1-0.3 \mathrm{~s}$. Figure 7 shows the two radio spectra indicated by white vertical lines in Figure 6. There are two narrowband radio bursts in the bottom panel at around 188.5 and $195.5 \mathrm{MHz}$; their bandwidths are about 2 and $1 \mathrm{MHz}$, respectively. The durations of these bursts are $100-$ $400 \mathrm{~ms}$, and they are not detected in the top panel observed after $250 \mathrm{~ms}$ from the bottom 


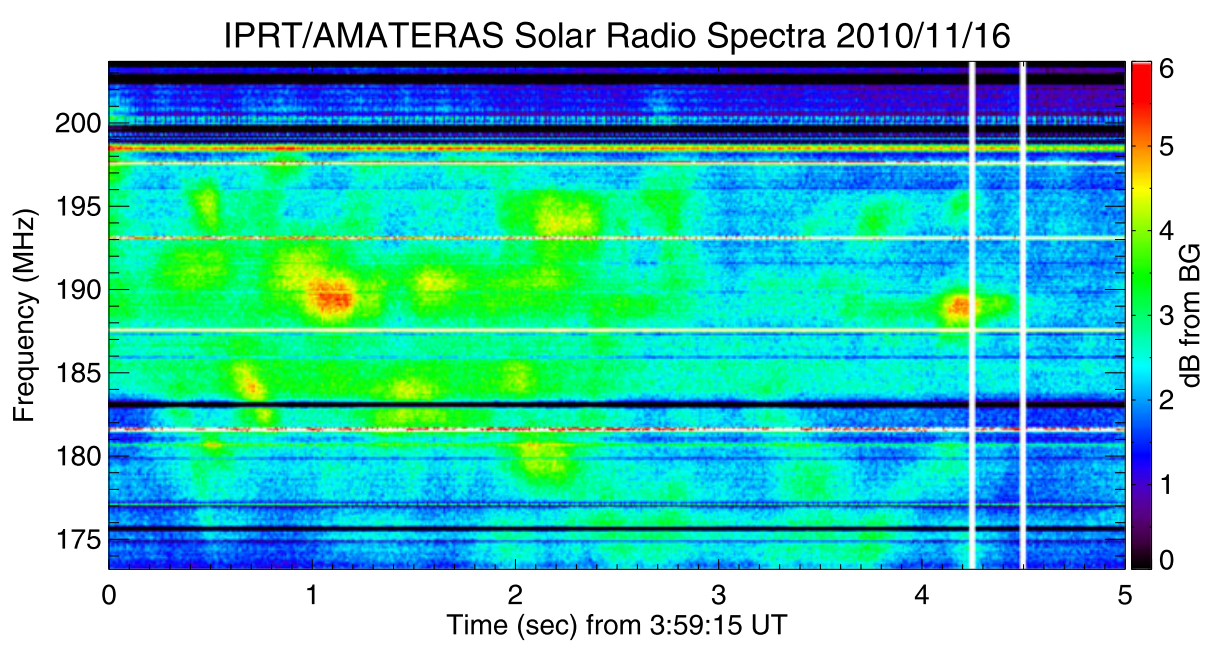

Figure 6 Zoomed-in image of spectrum indicated by white rectangle in Figure 5. Several horizontal lines in the dynamic spectra are radio frequency interferences (RFIs). The two spectra indicated by white vertical lines are shown in Figure 7.

Figure 7 Radio spectra of the bursts indicated by white lines in Figure 6: (top) observed at 3:59:19.500, (bottom) observed at $3: 59: 19.250$.
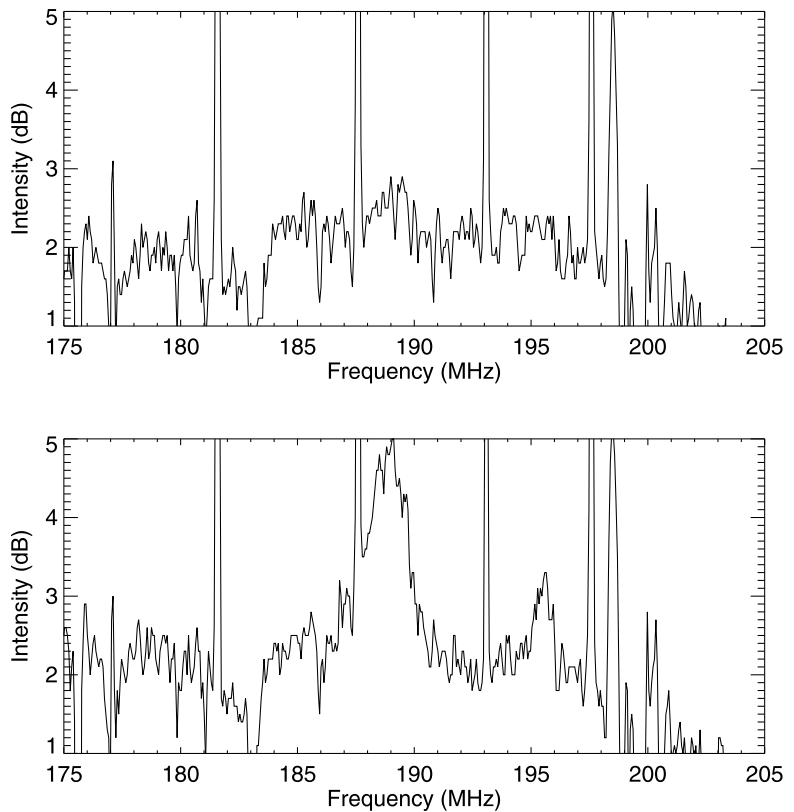

panel. These observation results show that AMATERAS can capture characteristics of the solar radio bursts that contain complex polarization variations and fine-spectrum structures. Detailed observations of the Type I noise storms from IPRT/AMATERAS are reported by Iwai et al. (2012). 


\subsection{Quality of Dynamic Spectrum}

It is generally difficult to separate fine-spectrum structures of the solar radio burst from artificial interference such as broadcasting or radiotelegraphy in frequency ranges that are allocated for common use. As the artificial interferences (RFIs) have a narrower bandwidth than the frequency resolutions of conventional frequency analyzers, frequency analyzers with wider frequency resolution are apt to be affected by RFIs in wider bandwidths. For example, in the case of the Iitate observatory, there are 20-30 artificial noise bands between 150 and $200 \mathrm{MHz}$ in the AMATERAS spectrum. When a frequency analyzer with a $1 \mathrm{MHz}$ bandwidth is adopted, it has 50 frequency channels from $150-200 \mathrm{MHz}$. This means that the total bandwidth contaminated by RFI is $20-30 \mathrm{MHz}$. On the other hand, the AMATERAS system has a narrower bandwidth of $61 \mathrm{kHz}$; hence, the total contaminated bandwidth is limited to less than $2 \mathrm{MHz}$. This enables a larger number of noise-free frequency channels to be obtained in the noisy frequency bands (see Figure 6). The large aperture also contributes to improving the spectrum quality, since some weak artificial interferences become weaker than the quiet Sun.

\section{Database}

The AMATERAS observational data are made public via the Inter-university Upper atmosphere Global Observation NETwork (IUGONET) project, ${ }^{2}$ which has developed a metadata database for various kinds of solar-terrestrial sciences.

Two kinds of data are provided in the database: low- and high-resolution data. The lowresolution data are archived in standard FITS formatted files. The dynamic range of the power spectrum is transformed from a 36-bit linear scale to an 8-bit log scale. Time and frequency resolutions of the low-resolution data are reduced to $1 \mathrm{sec}$ and $1 \mathrm{MHz}$, respectively. A quick look plot is also made of the low-resolution data. The FITS files and quick look plots are disclosed under the web page of the IPRT data center ${ }^{3}$ in the evening of each observation day. The high-resolution data set has the full time and frequency resolutions of the observation system. High-resolution data are archived in an 8-bit log scale only when some radio events are detected in the quick look plot. If some strong radio events are detected whose maximum intensity is higher than that of the quiet Sun by $25 \mathrm{~dB}$, high-resolution data are archived as a 16-bit log scale. The data rate of the high-resolution data is about $2500 \mathrm{MB}$ per hour if the data are archived in 8 bits.

\section{Concluding Remarks}

We have developed a new solar radio observation system in the litate observatory of Tohoku University, Japan. The developed system, named AMATERAS, is a spectropolarimeter consisting of a large aperture telescope, superheterodyne receiver system, and real-time digital analyzing system. The system has been calibrated using radio flux calibration sources. It enables us to observe solar radio bursts in the frequency range between 150 and $500 \mathrm{MHz}$. The minimum detectable flux in this frequency range is less than $0.7 \mathrm{SFU}$ with $10 \mathrm{~ms}$ accumulation time and $61 \mathrm{kHz}$ frequency bandwidth. Both left and right polarization components are

\footnotetext{
${ }^{2}$ http://www.iugonet.org/en/.

${ }^{3}$ http://pparc.gp.tohoku.ac.jp/data/iprt/.
} 
observed simultaneously. The observational data are archived soon after the daily observation and then made available on-line with their quick look figures.

The observational data will be used to analyze fine-spectrum structures of the metric solar radio burst to aid in understanding particle acceleration, propagation, and many other plasma processes in the solar corona. There are many other solar projects such as Hinode, STEREO, and SDO that provide observations in the X-ray, EUV, and optical ranges. Coordinated observations between the AMATERAS project and these presently operating and future observatories will be beneficial to the field of solar physics.

Acknowledgements We thank Satoshi Masuda and Yoshizumi Miyoshi for valuable discussions. Development of the AMATERAS system is supported by the Special Educational Research Budget from the Ministry of Education, Culture, Sports, Science and Technology (MEXT), Japan. This work was carried out by a joint research program of the Solar-Terrestrial Environment Laboratory, Nagoya University. K.I. was supported by a Japan Society for the Promotion of Science (JSPS) Research Fellowship for Young Scientists. We dedicate this paper to the victims of the Tohoku earthquake and wish for a speedy recovery of Fukushima prefecture.

Open Access This article is distributed under the terms of the Creative Commons Attribution Noncommercial License which permits any noncommercial use, distribution, and reproduction in any medium, provided the original author(s) and source are credited.

\section{References}

Aurass, H., Klein, K.-L., Zlotnik, E.Y., Zaitsev, V.V.: 2003, Astron. Astrophys. 410, 1001.

Baars, J.W.M., Genzel, R., Pauliny-Toth, I.I.K., Witzel, A.: 1977, Astron. Astrophys. 61, 99.

Benz, A.O., Messmer, P., Monstein, C.: 2001, Astron. Astrophys. 366, 326.

Benz, A.O., Grigis, P.C., Hungerbühler, V., Meyer, H., Monstein, C., Stuber, B., Zardet, D.: 2005, Astron. Astrophys. 442, 767.

Benz, A.O., Monstein, C., Meyer, H., Manoharan, P.K., Ramesh, R., Altyntsev, A., Lara, A., Paez, J., Cho, K.-S.: 2009a, Earth Moon Planets 104, 277.

Benz, A.O., Monstein, C., Beverland, M., Meyer, H., Stuber, B.: 2009b, Solar Phys. 260, 375.

Chernov, G.P.: 2006, Space Sci. Rev. 127, 195.

Güdel, M., Benz, A.O.: 1990, Astron. Astrophys. 231, 202.

Iwai, K., Miyoshi, Y., Masuda, S., Shimojo, M., Shiota, D., Inoue, S., Tsuchiya, F., Morioka, A., Misawa, H.: 2012, Astrophys. J. 744, 167.

Kondo, T., Isobe, T., Igi, S., Watari, S., Tokumaru, M.: 1995, J. Commun. Res. Lab. 42, 111.

Kontogeorgos, A., Tsitsipis, P., Caroubalos, C., Moussas, X., Preka-Papadema, P., Hilaris, A., et al.: 2006, Exp. Astron. 21, 41.

Kraus, J.D.: 1986, Radio Astronomy, 2nd edn., Cygnus-Quasar Books, Powell.

Magdalenic, J., Vršnak, B., Zlobec, P., Hillaris, A., Messerotti, M.: 2006, Astrophys. J. Lett. 642 , L77.

Mann, G., Aurass, H., Voigt, W., Paschke, J.: 1992. In: Proceedings of the First SOHO Workshop: Coronal Streamers, Coronal Loops, and Coronal and Solar Wind Composition SP-348, ESA, Noordwijk, 129.

McLean, D.J., Labrum, N.R.: 1985, Solar Radiophysics, Cambridge University Press, Cambridge.

Messmer, P., Benz, A.O., Monstein, C.: 1999, Solar Phys. 187, 335.

Prestage, N.P., Luckhurst, R.G., Paterson, B.R., Bevins, C.S., Yuile, C.G.: 1994, Solar Phys. 150, 393.

Sundaram, G.A.S., Subramanian, K.R.: 2005, Mon. Not. Roy. Astron. Soc. 359, 580.

Tsuchiya, F., Misawa, H., Imai, K., Morioka, A., Kondo, T.: 2010, Adv. Geosci. 19, 601. 